\section{Local Transmission of SARS-CoV-2 Lineage B.1.1.7, Brazil, December 2020}

\author{
Ingra Morales Claro, ${ }^{1}$ Flavia Cristina da Silva Sales, \\ Mariana Severo Ramundo, Darlan S. Candido, \\ Camila A.M. Silva, Jaqueline Goes de Jesus, \\ Erika R. Manuli, Cristina Mendes de Oliveira, \\ Luciano Scarpelli, Gustavo Campana, \\ Oliver G. Pybus, Ester Cerdeira Sabino, ${ }^{2}$ \\ Nuno Rodrigues Faria, ${ }^{2}$ José Eduardo Levi ${ }^{2}$
}

Author affiliations: University of São Paulo, São Paulo, Brazil (I.M. Claro, F.C.S. Sales, M.S. Ramundo, D.S. Candido,

C.A.M. Silva, J.G. de Jesus, E.R. Manuli, E.C. Sabino, N.R. Faria, J.E. Levi); University of Oxford, Oxford, UK (D.S. Candido, O.G. Pybus, N.R. Faria); Diagnósticos da América SA (DASA), Baueri, Brazil (C.M. de Oliveira, L. Scarpelli, G. Campana, J.E. Levi); Imperial College London, London, UK (N.R. Faria)

DOI: https://doi.org/10.3201/eid2703.210038

In December 2020, research surveillance detected the B.1.1.7 lineage of severe acute respiratory syndrome coronavirus 2 in São Paulo, Brazil. Rapid genomic sequencing and phylogenetic analysis revealed 2 distinct introductions of the lineage. One patient reported no international travel. There may be more infections with this lineage in Brazil than reported.

Cenomic sequencing and analysis during the seTvere acute respiratory syndrome coronavirus 2 (SARS-CoV-2) pandemic have led to identification of $\approx 800$ distinct SARS-CoV-2 lineages worldwide. A new phylogenetic cluster, B.1.1.7 lineage or variant of concern 202012/01, is characterized by 17 unique mutations and was first detected in southeastern England in late September 2020 (A. Rambaut et al., unpub. data, https://virological.org/t/ preliminary-genomic-characterisation-of-an-emergent-sars-cov-2-lineage-in-theuk-defined-by-a-novel-set-of-spike-mutations/563). As of January 17, 2021, this lineage had been confirmed in 38 countries (https://cov-lineages.org/global_ report_B.1.1.7.html). Epidemiologic and phylogenetic studies suggest that the rapid epidemic growth of B.1.1.7 in the United Kingdom is caused by its higher transmissibility (E. Volz et al., unpub. data, https://www.medrxiv.org/content/10.1101/2020.12.30.20249034v2; N. Davies, unpub. data, https://cmmid.github.io/topics/ covid19/uk-novel-variant.html), which could lead to increased incidence and higher peaks in hospitalizations

${ }^{1}$ These first authors contributed equally to this article.

${ }^{2}$ These senior authors contributed equally to this article. and deaths (N. Davies, unpub. data, https://cmmid. github.io/topics/covid19/uk-novel-variant.html).

We confirm 2 cases of infection with SARSCoV-2 B.1.1.7 lineage in Latin America. On December 30, 2020, we received saliva samples from 2 patients for genomic sequencing as part of research surveillance activities. Patient 1 was a woman 20 30 years of age residing in São Paulo, Brazil, who reported no travel outside of Brazil. Her symptoms began on December 21, and testing was conducted the next day. Patient 2 was a man 30-40 years of age who was tested in São Paulo on December 22 after having traveled from London on December 19. Ethics approval for this study was confirmed by the national ethics review board (Comissão Nacional de Ética em Pesquisa, protocol no. CAAE 30127020.0.0000.0068).

PCR testing (TaqPath COVID-19 PCR; ThermoFisher Scientific, https://www.thermofisher.com) performed as previously described (1) indicated that patient 1 was positive for the open reading frame $1 \mathrm{ab}$ (cycle threshold $\left.\left[C_{t}\right] 25.8\right)$ and nucleoprotein $\left(C_{t} 24.5\right)$ gene targets and patient 2 was positive for open reading frame $1 \mathrm{ab}\left(\mathrm{C}_{t} 28.1\right)$ and nucleoprotein $\left(C_{t} 27.29\right)$, but both were negative for the spike gene target. The 2 spike-gene dropout samples were identified among 400 samples collected during November 4-December 25, 2020.

For each sample, we conducted nanopore sequencing in duplicate by using the ARTIC protocol (https:/ / www.protocols.io/view/ncov-2019-sequencing-protocol-bbmuik6w). Concentrations of double-stranded DNA for the library-negative controls were below detection levels, indicating no contamination. We conducted whole-genome sequencing of SARS-CoV-2 by using the MinION platform (Oxford Nanopore Technologies, https://nanoporetech.com). By December 31 , sequencing statistics revealed 56,565 mapped reads for patient 1 and 51,761 for patient 2. Consequently, 28,023 bases for patient 1 and 26,339 for patient 2 were covered at $>25 \times$ depth. Consensus sequences covered $92.4 \%$ of the Wuhan Hu-1 reference genome (GenBank accession no. MN908947.3) for patient 1 and $87.1 \%$ for patient 2 . For the 2 newly generated genome sequences, we identified the B.1.1.7 lineage (assignment probability $=1.0$ ) by using the pangolin COVID-19 Lineage Assigner version 2.1.6 (2) (https://pangolin.cog-uk. io). The 2 genomes were made publicly available on GISAID (http://www.gisaid.org) on December 31, 2020 (identification nos. EPI_ISL_754236 for patient 1 and EPI_ISL_754237 for patient 2).

We next estimated a rapid maximum-likelihood phylogenetic tree $(3,4)$ for a multiple sequence align- 
ment (5) with the new sequences and 127 publicly available B.1.1.7 genomes from around the world available on GISAID (6) as of December 31, 2020 (https://github.com/CADDE-CENTRE/VOCLineage-Brazil). The virus genome recovered from patient 1 grouped within a well-supported cluster (bootstrap $85 \%$ ) of 10 sequences (60\% from the United Kingdom) (Figure). This finding is consistent with the travel history of an asymptomatic family member who was positive for SARS-CoV-2 (according to a rapid test performed on December 23, 2020), who arrived in São Paulo on December 17 after traveling from Italy to the United Kingdom and, after a short stay, from London to São Paulo, and who was in close contact with patient 1 . The sequence from patient 2 clustered with good statistical support (bootstrap 79.4\%) with a sequence collected in the United Kingdom on November 27. Patient 2 had traveled from London to São
Paulo on December 19 and was symptomatic when saliva was collected on December 22. Phylogenetic analysis suggests that this infection represents a second, independent introduction of the B.1.1.7 lineage from the United Kingdom to Brazil; patient 2 was not epidemiologically linked to patient 1.

Because information about this lineage from locations outside the United Kingdom is limited, our interpretations based on phylogenetic data might be biased by the different numbers of available genome sequences shared around the globe. Moreover, the samples that we analyzed were selected from only 2 cases confirmed by reverse transcription PCR in São Paulo; thus, our genomes were obtained from a small fraction of targeted spike-gene failure, and frequency of detection in our nonrandom sample does not represent prevalence of this lineage at the population level.



Figure. Phylogenetic context of novel severe acute respiratory syndrome coronavirus 2 B.1.1.7 genomes isolated from 2 patients in Brazil (labeled on figure), December 2020. Downsampling for the phylogenetic analysis of the B.1.1.7 SARS-CoV-2 variant $(n=4,693$, December 31,2020 ) was performed by selecting 1 sequence per country per day. As outgroups, we included 2 B.1.1 sequences from the United Kingdom that were closely related to the lineage of interest and sequence WH04 from Wuhan, China (GISAID identification no. EPI_ISL_406801; http://www. gisaid.org). Details on multiple alignment and phylogenetic tree reconstruction are described elsewhere (4). Tree file, aligned sequences, and GISAID acknowledgment tables are available at https://github.com/ CADDE-CENTRE/VOC-LineageBrazil. Scale bar indicates nucleotide substitutions per site. VOC, variant of concern. 
Despite temporary suspension of all flights to or from Brazil from or through the United Kingdom as of December 25, 2020 (http:/ / www.gov.uk/foreigntravel-advice/brazil), it is likely that the number of SARS-CoV-2 lineage B.1.1.7 infections in Brazil is higher than that reported. Increasing genomic surveillance of B.1.1.7 and other variants of concern that carry mutations of potential biological significance (e.g., E484K in the spike protein; C.M. Voloch, unpub data, https://www.medrxiv.org/content/10.1 $101 / 2020.12 .23 .20248598 \mathrm{v} 1$ ) is imperative for monitoring vaccination effectiveness and contextualizing the epidemiology and evolution of SARS-CoV-2 in Latin America.

\section{Acknowledgments}

We thank all researchers who are working around the clock to generate and share genome data worldwide on GISAID (http://www.gisaid.org). GISAID acknowledgment tables are available at https:/ / github.com/CADDE-CENTRE/ VOC-Lineage-Brazil.

This project was supported by a Medical Research Council-São Paulo Research Foundation (FAPESP) CADDE partnership award (MR/S0195/1 and FAPESP 18/14389-0) (http:// caddecentre.org/). N.R.F. is supported by a Wellcome Trust and Royal Society Sir Henry Dale Fellowship (204311/Z/16/Z).

\section{About the Author}

Mrs. Claro is a PhD student at the Department of Infectious Disease, School of Medicine \& Institute of Tropical Medicine, University of São Paulo, São, Paulo, Brazil. She is a part of the Centre for Arbovirus Discovery, Diagnostics, Genomics and Epidemiology team and has primary research interests in real-time epidemiologic surveillance of viruses of public importance for Brazil.

\section{References}

1. Vogels CBF, Watkins AE, Harden CA, Brackney DE, Shafer J, Wang J, et al. SalivaDirect: a simplified and flexible platform to enhance SARS-CoV-2 testing capacity. Med. 2020 Dec 26 [Epub ahead of print]. https://doi.org/10.1016/j. medj.2020.12.010

2. Rambaut A, Holmes EC, O'Toole Á, Hill V, McCrone JT, Ruis $\mathrm{C}$, et al. A dynamic nomenclature proposal for SARS-CoV-2 lineages to assist genomic epidemiology. Nat Microbiol. 2020;5:1403-7. https:/ / doi.org/10.1038/ s41564-020-0770-5

3. Minh BQ, Schmidt HA, Chernomor O, Schrempf D, Woodhams MD, von Haeseler A, et al. IQ-TREE 2: new models and efficient methods for phylogenetic inference in the genomic era. Mol Biol Evol. 2020;37:1530-4. https://doi.org/10.1093/molbev/msaa015

4. Candido DS, Claro IM, de Jesus JG, Souza WM, Moreira FRR, Dellicour S, et al. Evolution and epidemic spread of SARS-
CoV-2 in Brazil. Science. 2020;369:1255-60. https://doi.org/10.1126/science.abd2161

5. Katoh K, Standley DM. MAFFT multiple sequence alignment software version 7: improvements in performance and usability. Mol Biol Evol. 2013;30:772-80. https://doi.org/10.1093/molbev/mst010

6. Shu Y, McCauley J. GISAID: Global Initiative on Sharing All Influenza Data - from vision to reality. Euro Surveill. 2017;30;22:30494.

Address for correspondence: Nuno R. Faria, St Mary's Hospital, Praed St, Paddington, London W2 1NY, UK; email: nfaria@ ic.ac.uk; and José Eduardo Levi, Avenida Juruá 548, Alphaville, Barueri, SP 06455-010, Brazil; email: jose.levi.ext@dasa.com.br

\section{Mycobacterium bovis Pulmonary Tuberculosis, Algeria}

\author{
Fatah Tazerart, Jamal Saad, Abdellatif Niar, Naima \\ Sahraoui, ${ }^{1}$ Michel Drancourt ${ }^{1}$
}

Author affiliations: Université Ibn Khaldoun de Tiaret, Tiaret, Algeria (F. Tazerart); Université de Blida, Blida, Algeria (F. Tazerart, N. Sahraoui); Institut Hospitalo-Universitaire Méditerranée Infection, Marseille, France (F. Tazerart, J. Saad, M. Drancourt); Aix-Marseille-University, Marseille (J. Saad, M. Drancourt); Laboratoire de Reproduction des Animaux de la Ferme, Université Ibn Khaldoun de Tiaret, Tiaret (A. Niar)

DOI: https://doi.org/10.3201/eid2703.191823

We analyzed 98 Mycobacterium tuberculosis complex isolates collected in 2 regions of Algeria in 2015-2018 from 93 cases of pulmonary tuberculosis. We identified 93/98 isolates as $M$. tuberculosis lineage 4 and 1 isolate as $M$. tuberculosis lineage 2 (Beijing). We confirmed 4 isolates as $M$. bovis by whole-genome sequencing.

Tn Algeria, interpreting tuberculosis (TB) incidence, Lestimated at $53-88$ cases $/ 100,000$ population in 2017 (1), is limited by the fact that the diagnosis relies on microscopic examination of clinical samples. Iso-

${ }^{1}$ These authors equally contributed to this work. 